\title{
Electrical charging effects on the sliding friction of a model nano-confined ionic liquid
}

\author{
R. Capozza ${ }^{1,2}$, A. Benassi ${ }^{2,3}$, A. Vanossi ${ }^{2,1}$, and E. Tosatti ${ }^{1,2,4}$ \\ 1 International School for Advanced Studies (SISSA), Via Bonomea 265, 34136 Trieste, Italy \\ ${ }^{2}$ CNR-IOM Democritos National Simulation Center, Via Bonomea 265, 34136 Trieste, Italy \\ 3 Institute for Materials Science and Max Bergmann Center of Biomaterials, TU Dresden, 01062 Dresden, Germany \\ ${ }^{4}$ International Centre for Theoretical Physics (ICTP), Strada Costiera 11, 34014 Trieste, Italy
}

(Dated: August 18, 2015)

\begin{abstract}
Recent measurements suggest the possibility to exploit ionic liquids (ILs) as smart lubricants for nano-contacts, tuning their tribological and rheological properties by charging the sliding interfaces. Following our earlier theoretical study of charging effects on nanoscale confinement and squeezout of a model IL, we present here molecular dynamics simulations of the frictional and lubrication properties of that model under charging conditions. First we describe the case when two equally charged plates slide while being held together to a confinement distance of a few molecular layers. The shear sliding stress is found to rise strongly and discontinuously as the number of IL layers decreases stepwise. However the shear stress shows, within each given number of layers, only a weak dependence upon the precise value of the normal load, a result in agreement with data extracted from recent experiments. We subsequently describe the case of opposite charging of the sliding plates, and follow the shear stress when the charging is slowly and adiabatically reversed in the course of time, under fixed load. Despite the fixed load, the number and structure of the confined IL layers changes with changing charge, and that in turn drives strong friction variations. The latter involve first of all charging-induced freezing of the IL film, followed by a discharging-induced melting, both made possible by the nanoscale confinement. Another mechanism for charging-induced frictional changes is a shift of the plane of maximum shear from mid-film to the plate-film interface, and viceversa. While these occurrences and results invariably depend upon the parameters of the model IL and upon its specific interaction with the plates, the present study helps identifying a variety of possible behavior, obtained under very simple assumptions, while connecting it to an underlying equilibrium thermodynamics picture.
\end{abstract}

PACS numbers: 68.35.Af,68.08.De,62.10.+s,62.20.Qp

\section{INTRODUCTION}

Ionic liquids (ILs) - organic salts that are liquid at room temperature - are of considerable and increasing physical and technological interest. ILs are characterized by negligible vapor pressures, high temperature stability and a wide electrochemical window [1]. Moreover their physical properties can be widely tuned by changing the molecular structure of the cation-anion pairs 2. Many ILs strongly adhere to solid surfaces 3 and can form wear-protective films capable of resisting much higher loads than those of molecular lubricants [4]. For this reason among others their use as boundary lubricants has been recently pursued in experimental studies under nanoscale confinement, employing both the surface force apparatus (SFA) [4, 5, and atomic force microscopy $(\mathrm{AFM})$ 6 10, as well in in a number of computer simulations at various levels of idealization 11-13. Our specific interest focuses here on the exploration of the frictional changes induced by electrical charging of the plates which confine the IL, a subject only partially covered by previous theoretical works [13 15. Besides lubrication, the dynamical behavior of ILs under charging is of additional interest in the field of supercapacitors [16, 17. Experimentally, there is a number of nano-frictional studies of surfaces charged with respect to a reference electrode [8, 9, 18. Not so between two oppositely charged electrodes. Recent macroscopic friction studies have begun to appear for IL sliding under charging conditions [19, 20]. Here however we shall restrict ourselves to friction under conditions of nanoscale confinement.

At the molecular level, ILs confined between hard plates become structured in the form of layers that run parallel to the plates, not unlike other liquids but further characterized by the charge order typical of molten salts, with an alternation of positive and negative ion layers, and an interlayer separation that corresponds to the ion pair size [4, 6, 10, 21 27]. Plate charging is expected to give rise to rearrangements of the IL layering and ordering, at least near the confining plate-IL interfaces, with a consequent and probable change of the lubrication properties under plate sliding. Charging-induced friction changes have already been to some extent detected and described experimentally [8, 9]. Not unexpectedly, they are reported to depend on the particular kind of IL and of confining surfaces chosen. For example, a strong reduction of the friction of an AFM silica colloid probe was found by negatively biasing a $\mathrm{Au}(111)$ surface 8 immersed in $\left[P y_{1,4} F A P\right]$. However, a similarly strong friction drop occurred at positive bias upon sliding a sharp AFM tip on highly oriented pyrolytic graphite immersed in a $[H M I m] F A P$ IL $[9]$.

This diversity of behavior, reflecting the variety of ILs and the different nature of the confining and sliding sur- 
faces, suggests some flexibility in the corresponding theoretical modeling, aimed at a broad exploration of how some of this variety of behavior could be addressed with a handful of model parameters, rather than describing in details one or another particular case. The minimal IL model and a natural first choice is a simple molten salt, such as liquid $\mathrm{NaCl}$. Fedorov and Kornyshev [14 did that, their model consisting of two spherical LennardJones (LJ) particles with unequal radii further endowed with opposite charges. The frictional behavior of that model was recently investigated by Fajardo et al. 13. who noted interesting similarities with data by $\mathrm{Li}$ et al. 9. However, a parallel study which we conducted of this type of model under simple confinement and squeezout 15] revealed that the confined liquid film evolved far too sharply from a layered liquid for neutral plates at large spacings, to a strongly crystallized rocksalt structure solid under narrow confinement and/or plate charging. On top of that, the simple molten salt model wetted the plates much too readily, even given a reasonable choice of parameters. Both of these aspects, excessive crystallization and excessive wetting tendencies, represent a severe oversimplification by comparison with real IL lubricants whose wetting capability is generally more modest, and where, owing to far more complex molecular structures, the layering and solidification which occurs under strong confinement does not usually imply cystallization, and is replaced by glass-like disordered structures. That complex behavior is of course much better captured by fully realistic IL model simulations, of which there are several good examples in literature, [11, 12] but whose difficulty and intrinsic complexity make the studies much more work-intensive while at the same time emphasizing aspects that are to some extent undesirably specific to each case.

A previous study of our own IL model [15] was designed to bridge the gap between these two extremes, either oversimplification or overspecificity. We showed that a minimal modification of the charged LJ model, consisting of a neutral "tail" rigidly attached to one of the ions (the cation in our case, but the anion could have been equivalently chosen) could go a long way in our desired direction, making the structural and squeezout properties of the confined IL model considerably more realistic while still not specific. While the anion and the charged part of the cation retain a strong Coulomb correlation, the neutral tail is generally and merely pushed out of the way, resulting in poor tail-tail correlations. The tail-induced disorder, along with the steric hindrance they introduce, impede overall rocksalt crystallization with drastic changes in the ILs solidification, also introducing new unexpected structures as well as the desired glassy aspects and wettability features. These structural features were found to evolve and change under plate charging, foreshadowing a variability that could be explored through a variation of the model parameters. At the same time, this IL "tailed model" (TM), to be further detailed below, is simple enough to permit a novel, quantitative calculation and characterization of the confined IL enthalpy versus interplate distance, and - of crucial importance here its variations under plate charging. The minima of the enthalpy-distance curves identify stable or metastable layering states of the confined IL, showing a perfect fit with the evolution in the structural layering oscillations of density and charge observed in simulations of the confined IL under variable interplate separation and plate charge. The layering transitions during squeezout, well established in real IL experiments [5, 22, 28, are reproduced by the TM model and directly related to jumps between enthalpy minima, while these minima evolve and cross values under closing of the gap between plates, or under variable plate charging [15. With that very useful characterization, the TM model provides a ready tool for a fresh study of some generic effects of electrical plate charging on the nanoscale sliding friction of confined ILs.

In this work we first introduce and detail the TM model and the chosen confining geometry. We then analyze the sliding simulations and discuss how the structural changes, induced by a negative plate charging, modify the frictional properties. Interesting observations emerge examining the shear velocity profile inside the IL film as a function of the plate charging. First, we observe a switching of the shear band plane from the plate-film interface, typical of low friction, to the mid-film region, typical of larger friction. A second important effect is the charging-induced solidification/melting process described in our previous, static study, and typically ignored in literature. Charged plates generally increase the IL wetting, giving rise to "electrowetting". But, as most ILs are not far above freezing at room temperature, increased wetting will in turn encourage solidification, the capability to withstand load, with a large effect on friction. Conversely, plate neutrality and partial dewetting facilitates melting of the IL film, leading to squeezout under even moderate load.

The final and most specific part of this work is the investigation of the effects on friction of opposite charging (equivalent to an applied interplate voltage) of the two confining plates. A given charge state of the plates is accompanied by a strong structuring of the IL near and between the plates. A reversal of plate charges is followed by an accompanying destructuring and even melting of that layering structure, prior to the reversal of local IL ordering and eventual re-solidification. The sliding friction between plates undergoes a corresponding chargedependent evolution as a consequence. This kind of phenomena, only poorly explored so far, constitute a fresh prediction of this study. 

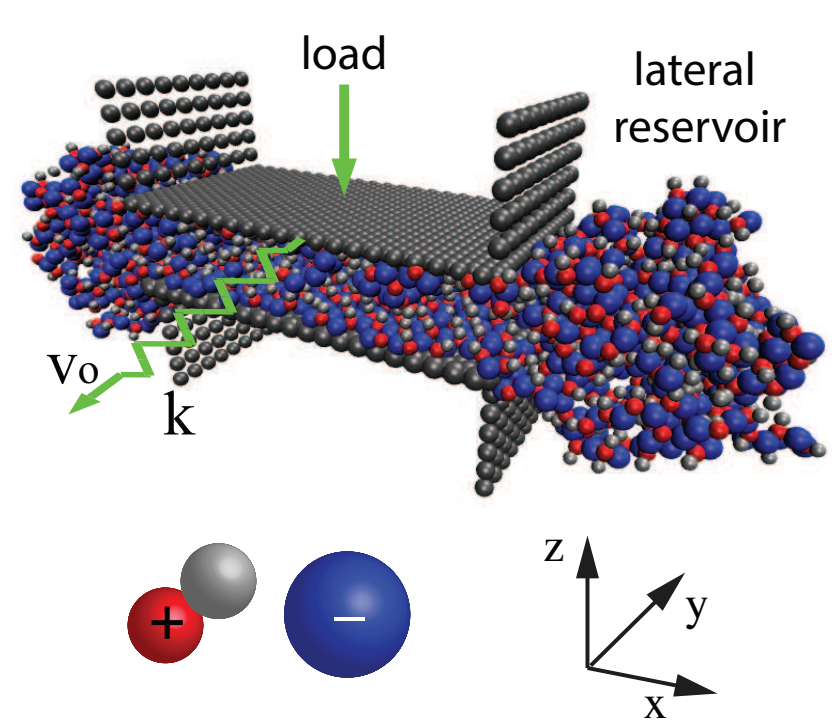

FIG. 1: (Color online) Sketch of the simulation geometry with open boundaries along $x$ and $z$ directions and periodic boundaries along $y$, i.e. the direction along which the sliding force is applied through a spring moving with speed $V$, a situation conceptually similar to an SFA setup. The width $L$ of the plates along $x$ is $20 \mathrm{~nm}$. By applying an external $z$-oriented force to the top plate, both at $V=0$ and at $V>0$, the size $D$ of the gap between the plates can change since the liquid can flow in and out the lateral reservoirs. The shapes of the anion (blue) and cation (red) are sketched in the lower left corner.

\section{MODEL, SIMULATION DETAILS, SYSTEM PREPARATION}

To pursue our desired broad scenario of possible charging effects on the lubrication properties of nanoconfined ILs we adopt the simple TM model 15 which deliberately neglects the fine structure of the ions retaining only minimal features common to most of the ILs, such as the anion-cation asymmetry, their possible amphiphilic character and their generally irregular shape. The TM model is a two-component charged Lennard-Jones (LJ) system where anions and cations not only have different radii but where a tail is attached to the cation. The cation is therefore a dimer consisting of a positively charged, small-sized LJ head, rigidly bound to an equal size, neutral particle, see Fig. 1. The effect of the tail upon the ILs wetting and confinement properties is important, as mentioned in the introduction and recently shown in ref. [15, where the details about the adopted potentials and the corresponding parameters are also specified and discussed. Essentially the tails give the molecules a larger gyration ratio in the liquid state, and prevent low temperature ordering replacing crystallization with a glass-like solidification - all features that are common and important in real ILs. All the molecular dynamics (MD) simulations were performed using the LAMMPS code 29. The long range Coulomb interactions have been treated in reciprocal space using a particle-particle particle-mesh solver. Canonical ensemble configurations were generated by means of a Langevin thermostat directly applied to the lubricant molecules. The plates were treated as rigid bodies, the lower one fixed and the upper one subjected to a $z$-directed load force $F_{N}$ and driven to slide along $(\mathrm{x}, \mathrm{y})$. Their structure consists of rigid close-packed triangular lattices of LJ particles with nearest neighbor spacing $0.52 \mathrm{~nm}$, mimicking the periodicity of a mica surface. A graphite, or graphene plate, which unlike mica may be electrically charged [9] has a periodicity of about $0.246 \mathrm{~nm}$, which is not too different from half that value. In most of our simulations the liquid is confined between two identical plates with a modest surface charge density $q=-4 \mu \mathrm{C} / \mathrm{cm}^{2}$, subsequently increased to $q=-12 \mu \mathrm{C} / \mathrm{cm}^{2}$. As a reference, the surface charge density quoted for mica in contact with $\mathrm{H}_{2} \mathrm{O}$ is $-33.4 \mu \mathrm{C} / \mathrm{cm}^{2}$, a much larger value 30 . The state of charge of a mica surface in contact with an IL will certainly differ, but it is still generally believed that some charging remains. Since nature as well as the algorithm used to treat the long range interaction in reciprocal space require the overall system to be neutral, when endowing both plates with a total net nonzero charge we correspondingly add extra ions of the opposite charge to compensate.

The lateral drive is actuated through a spring $k$ connected to the top plate and pulled at constant velocity $V$. The same value $V=2.2 \mathrm{~m} / \mathrm{s}$ was adopted throughout unless another value is explicitly stated. The instantaneous frictional force opposing the motion is measured through the elongation of the driving spring as $F_{L}(t)=k\left(V t-Y_{C M}(t)\right)$, where $Y_{C M}(t)$ is the $y$ coordinate of the top plate center of mass. The relevant quantity in friction is eventually the shear stress $\sigma=\left\langle F_{L}\right\rangle / A$ where $A$ is the area of the contact. In our case $A=177 \mathrm{~nm}^{2}$ is a constant, so presenting $<F_{L}>$ or $\sigma$ is just the same. In experiments however the contact area is not generally constant, so that the shear stress must be extracted before making comparisons.

The open geometry described in Fig. 1 has been chosen because it permits particles to be squeezed out or sucked in from two lateral IL droplets. These droplets thus serve as liquid reservoirs allowing the number of ions effectively confined inside the gap to adjust and change dynamically depending on the loading, charging, and sliding conditions, realizing a kind of effectively grandcanonical configuration. The distinguishing feature of the open boundary geometry employed here, as opposed to several previous studies which used closed periodic boundary conditions, is to address a situation much closer to real SFA or AFM experiments, permitting in particular squeezeout transitions and the consequent transverse $(z)$ and in-plane $(x, y)$ reordering. 
Before the sliding simulations begin, we prepared equilibrium starting configurations. In our protocol, the starting configurations were obtained by filling the open gap between initially distant plates, in any desired state of charge, with IL, and then reducing the gap width $D$ with $\dot{D}=0.11 \mathrm{~m} / \mathrm{s}$, generally slow enough to enact a reasonably adiabatic squeezout, as was found in Ref. [15. The adiabaticity is facilitated by our chosen temperature $T=225 K$, substantially higher the TM bulk melting temperature $T_{\text {melt }} \simeq 150 \mathrm{~K}$ [15], but as will be discussed below it is not automatically guaranteed, because both confinement and charging favor an increase of viscosity and a tendency to solidify. Above a minimal interplate distance $D_{c} \simeq 4 \mathrm{~nm}$ and for small plate charge, the simulated IL is completely liquid, even if structured in close vicinity of the plates owing to their rigid wall nature and also to their charging. Interestingly, this near-plate local structure develops not only along $z$, i.e., normal to the interface, but also along $(x, y)$, parallel to the interface. Parallel IL ordering, rather more difficult to detect experimentally, is currently being pursued and detected with AFM tools [31, 32]. As $D$ is further and gradually decreased, the confinement effects increase, the liquid-like diffusion diminishes and below a critical film thickness $D_{c}$ there is an effective interlocking of the structure emanating from the two facing plates, leading to a freezing of the IL into a solid-like layered arrangement with an odd number $N_{\text {layer }}$ of alternating charge layers - when the two plates are neutral or identically charged. While no claim can obviously be made to represent a realistic situation, we note that IL confinement between mica plates, typically used in SFA friction experiments, is known to behave precisely like that [4, 22].

As was shown in the previous paper [15] our choice of scheme and geometry permits additional thermodynamical insights. Treating the interplate separation $D$ as the external control variable, a free enthalpy $H\left(D, F_{N}, q\right)$ can be calculated first estimating the free energy $W(D)$ by integration of the average force $\langle F(D)>$ measured between the plates as their distance $D$ is reduced.

$$
W(D)=\int_{D}^{\infty}<F(z)>d z
$$

where $\langle F(z)>$ is the average z-oriented force exerted by the IL on the plates while they are a distance $z$ apart. After that the free enthalpy at given load and charge can be obtained as

$$
H\left(D, F_{N}, q\right)=W(D)+F_{N} D
$$

Free enthalpy curves are displayed in Fig 2 for two values of normal load $F_{N}$, and predict the load-induced transition $N_{\text {layer }} \rightarrow N_{\text {layer }}-2$ layers. The plate-plate free enthalpy generally displays as a function of $D$ several odd- $N$ minima whose relative values depend on plate charging and load. Once specified charge and load, one of them is the absolute minimum, therefore the thermodynamically stable layered state, and all others are higher, metastable states, which may still be very long lived. If the layered state is obtained by applying a force rather than by forcing a distance $D$, the precise number of layers attained in the course of a given simulation (or experiment) will be history-dependent, in that more than one observed thickness may correspond to the same applied force. This picture is quite close to what is observed in SFA and AFM [4, 5, 33. In addition to the alternating charge layer structure, we had previously found that the confinement-induced solid structure of the TM model, although far from completely cystalline, carried a second partly crystalline feature, consisting of vertical, neutral planes, inside which the anions and cations arrange in a roughly square lattice, albeit with totally disordered tail orientations [15]. Further decrease of $D$ caused this nearly solid IL to squeeze out by successive pairs of ion planar layers, positive and negative, thus preserving local charge neutrality, reducing the extension of vertical planes.

Starting with large $D$ and an initially fluid IL between the plates, the force $\langle F(D)>$ resisting squeezout, initially zero, rises as $D$ drops. The IL is partly squeezed out, and the part that remains trapped acquires a structure consisting of $N_{\text {layer }}$ layers (odd for equally charged plates, even for opposite charging). The squeezout transitions $N_{\text {layer }} \rightarrow N_{\text {layer }}-2$ are clear first order transitions in this picture, the free enthalpy jumping across barriers between successive $H(D)$ local minima. Decreasing $D$, and thus increasing $\langle F(D)>$ the lowest enthalpy state moves to a lower and lower number of layers. Each of these states with progressively lower, integer number of IL layers provides a starting state for our successive sliding simulations.

Upon checking, we actually found that configurations generated by fast squeezouts often possess internal layers that are only partly populated. This kind of ill-prepared states can survive as metastable for a long time. When faced with these ill-prepared states, initial particle configurations were further refined until a sufficiently reliable equilibrium particle population was reached. That allowed us to discard as a rule metastable initial configurations, and to describe the sliding behaviour of the stable, history independent ones, striving to discard metastable states as much as possible within our modest simulation durations.

\section{SIMULATED SLIDING}

As mentioned earlier, there are two types of plate charging relevant for sliding friction. The first type, and the one generally realized experimentally, is single-plate charging relative to an electrode in electrical contact with the IL. In this case the aspect that matters is the IL 


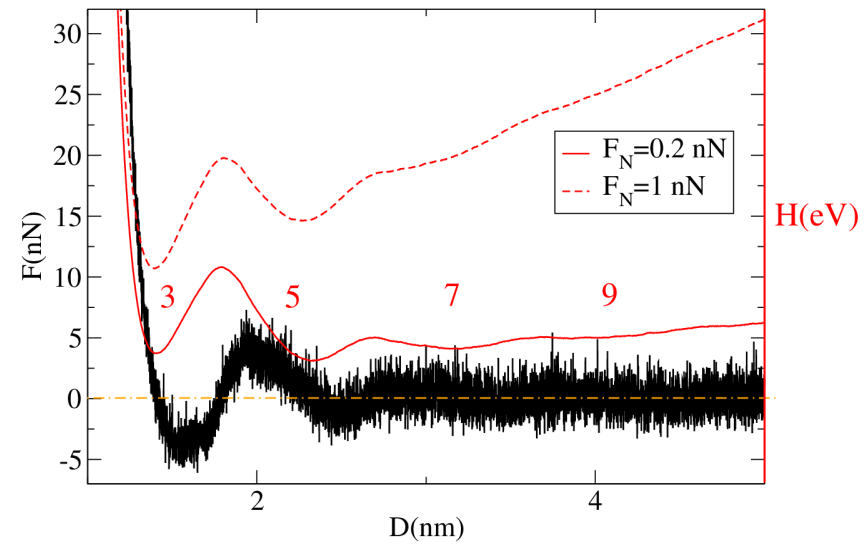

FIG. 2: (Color online) Interplate force $F(z)$ as a function of distance, and free enthalpy $H(D)$ calculated by Eq.(1) in the absence of sliding and for indicated values of normal load $F_{N}$ and an equal charge on both plates of $q=-4 \mu \mathrm{C} / \mathrm{cm}^{2}$.

structuring close to the charged plate, whereas the second plate, or tip, often insulating, acts as a merely mechanical probe of that structure. We replace this situation by simulating two equally charged plates, neutralized by adding the right amount of extra ions to the IL. The second type, whose experimental feasibility we ignore, but which looks even more interesting, is that of opposite plate charging. This we simulate without difficulty, restricting the charge magnitude to values small enough to correspond to an interplate voltage below $5 \mathrm{~V}$.

We start off our sliding simulations with equally charged plates, endowed with a negative charge density $q=-12 \mu \mathrm{C} / \mathrm{cm}^{2}$ (about $1 / 3$ of the nominal value quoted for mica in experimental conditions) and with the IL confined between them in a variety of $N_{\text {layer }}$ configurations, obtained as a result of squeezout down to different $D$ values, as detailed in the previous section, further equilibrated under an applied load $F_{N}$, chosen to be close to $<F(D)>$. Sliding friction depends upon the IL layer number $N_{\text {layer }}$ and the load $F_{N}$.

Fig 3 illustrates two sliding simulations starting from $N_{\text {layer }}=7$ with either $F_{N}=0.2 \mathrm{nN}$ and $F_{N}=0.9 \mathrm{nN}$. The simulated system at $F_{N}=0.2 \mathrm{nN}$ maintains 7 layers confined in between the plates, while that at $F_{N}=0.9 \mathrm{nN}$ undergoes a $7 \rightarrow 5$ relayering transition, whose kinetics seems favored by sliding.

As shown in Fig $3 \mathrm{~b}$, this transition corresponds to the sudden drop of the gap $D$, associated with the expulsion of the pair of layers, replacing the $N_{\text {layer }}=7$ metastable state with the stable $N_{\text {layer }}=5$. This is an example of how the lateral driving can facilitate the squeezout of layers by mechanically perturbing the IL.

The close proximity of the $7 \rightarrow 5$ transition can be predicted by free enthalpy curves, displayed in Fig 3 . At $F_{N}=0.9 \mathrm{nN}$ the energy barrier separating the states with 7 and 5 layers drops. The role of sliding appears
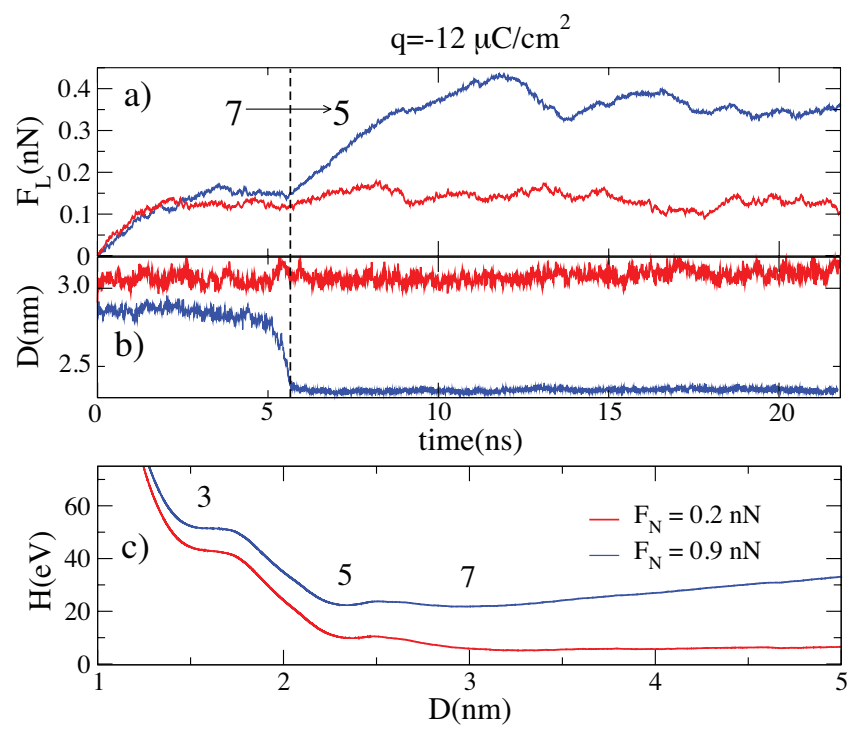

FIG. 3: Time evolution of a) the frictional force $\left\langle F_{L}\right\rangle$ and b) interplate gap width $D$ as a function of time for two normal loads $F_{N}=0.2 \mathrm{nN}$ (stable) and $F_{N}=0.9 \mathrm{nN}$ (metastable). c) Free enthalpy curves from squeezout before sliding for indicated values of $F_{N}$. Note a stronger local minimum at $N_{\text {layer }}=5$ for $F_{N}=0.9 \mathrm{nN}$, justifying the $7 \rightarrow 5$ transition under sliding.

to be kinetic, facilitating the jump to the more stable 5 layer state.

\section{$N_{\text {layer- }}$ DEPENDENT FRICTIONAL SHEAR STRESS}

The calculated force-distance curves of Ref. [15] show that a single normal load $F_{N}$ generally gives rise to several long lived states with a different number $N_{\text {layer }}$. For example $F_{N}=0.5 \mathrm{nN}$ can support $N_{\text {layer }}=3,5,7$ [15, very much as it happens in experiment [4, 10, 21]. Figure 4 shows the lateral force $\left\langle F_{L}>\right.$ obtained as a function of time for different $N_{\text {layer }}$ values, with $F_{N}=0.5 \mathrm{nN}$ and $q=-12 \mu \mathrm{C} / \mathrm{cm}^{2}$. Static free enthalpy curve in Fig $4 \mathrm{~b}$ demonstrates that $N_{\text {layer }}=7$ is stable configuration while 3 and 5 are metastable. A strong friction increase is observed with decreasing $N_{\text {layer }}$, in agreement with expectations and with SFA data [21, 23. The detailed load dependence of the frictional shear stress obtained for each fixed number of layers is more interesting, and is illustrated in Fig 5 a. Here the growth of shear stress with increasing load $F_{N}$ is surprisingly modest. While that might appear at first sight in disagreement with experiment, which show a nearly linear increase of kinetic friction with load [21, we must recall that the contact area is not a constant in SFA, whereas shear stresses require normalizing to the area $A$. Assuming for SFA two perpendicularly oriented elastic cylinders in Hertzian contact, the 

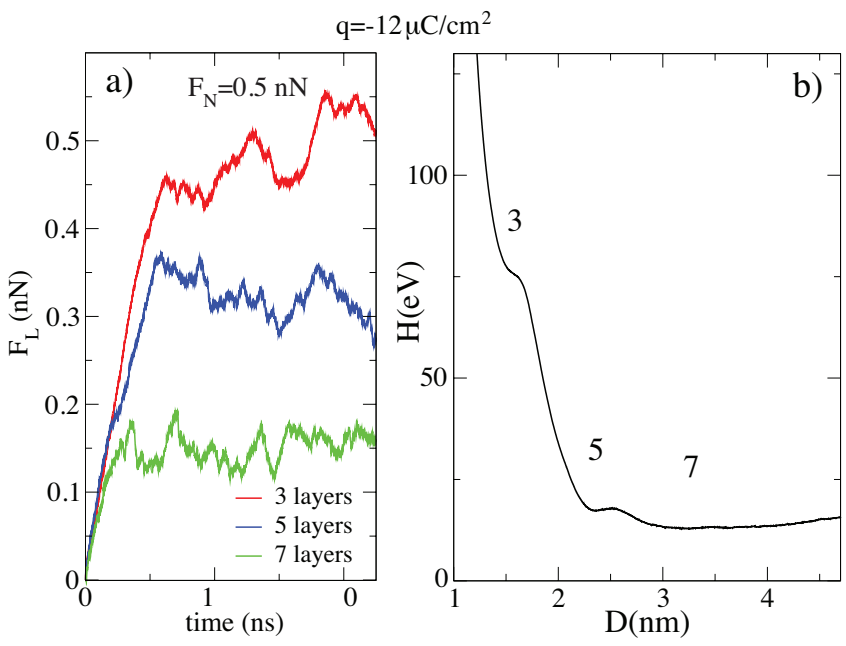

FIG. 4: a) Lateral force $\left\langle F_{L}\right\rangle$ as a function of time for plate charge density of $q=-12 \mu \mathrm{C} / \mathrm{cm}^{2}$ and for different number of confined layers $N_{\text {layer }}$ under the same load $F_{N}=0.5$. b) Static free enthalpy curve for $F_{N}=0.5 \mathrm{nN}$, showing that $N_{\text {layer }}=7$ is stable configuration while 3 and 5 are metastable ones.
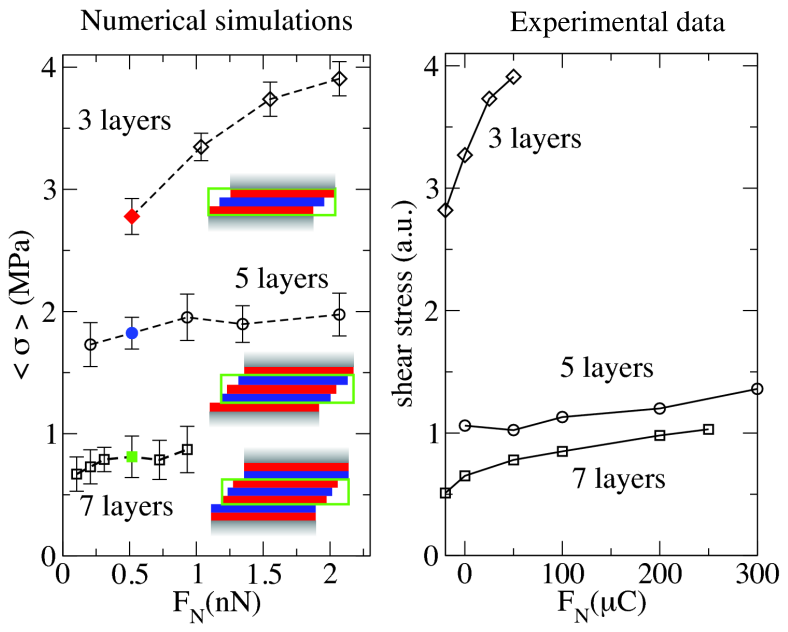

FIG. 5: a) Average shear frictional stress $\langle\sigma\rangle=\left\langle F_{L} / A\right\rangle$ as a function of load $F_{N}$ for the indicated number of confined layers and a charge density of $q=-12 \mu \mathrm{C} / \mathrm{cm}^{2}$ on both plates. The colored markers refer to the force profiles in panel a) of Fig 4. The cartoons suggest how, upon reducing the number of layers, the shear plane moves closer to interfaces, where particles are strongly bound to the plates. b) Approximate behaviour of the shear stress observed with [C4C1Pyrr][NTf2] confined between two mica sheets in a Surface Force Balance experiment. This plot has been obtained by rescaling kinetic friction force data from Ref. 21 by a contact area taken as $A \propto F_{T}^{2 / 3}$ (see text). Theoretical and experimental shear stresses agree remarkably well, showing a relatively modest increase with load.

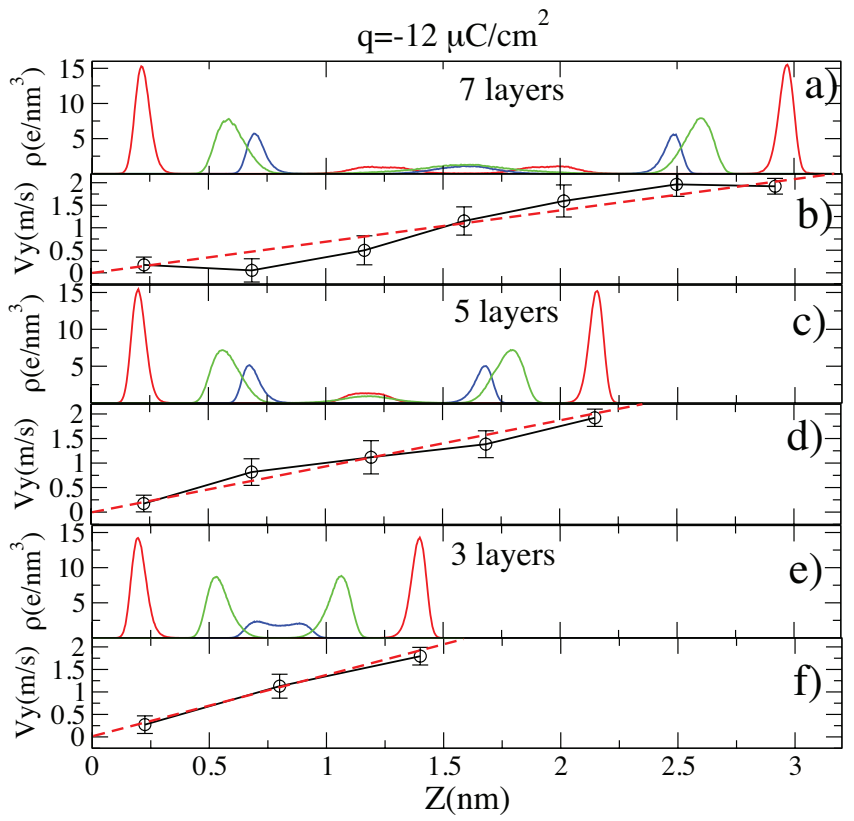

FIG. 6: Charge density $q=-12 \mu \mathrm{C} / \mathrm{cm}^{2}$ on both plates, top plate driven with velocity $V=2.2 \mathrm{~m} / \mathrm{s}$. Layer-by-layer density profiles ( (a), (c) and (e)) and sliding velocity ( (b), (d) and (f) ) relative to the bottom plate, for $N_{\text {layer }}=7, N_{\text {layer }}=5$ and $N_{\text {layer }}=3$ respectively. Red, blue and green curves represents cation, anion and tail densities respectively. Comparison with average expected velocities (dashed lines) shows that shear is maximal in the three inner layers (the "shear band"), moving closer to the plates as $N_{\text {layer }}$ decreases. Error bars in panels $\mathrm{b}, \mathrm{d}$, f have been reduced ten times for clarity.

area $A \propto F_{T}{ }^{2 / 3}$, where $F_{T}=F_{\text {adhesion }}+F_{N}$ is the effective force between the two sliders. Extracting an approximate adhesion force by linearly extrapolating the experimental kinetic friction to zero in Fig.1 of Ref. [21] , we recover the "experimentally derived" shear stress vs load curves in Fig 5b. Remarkably, they now show a weaker experimental dependence on load, actually very close to that predicted by simulations in Fig. 5 a.

To understand the strong dependence of $\left\langle F_{L}\right\rangle$ on the number of layers illustrated in Fig 4 a, we now take a closer look to the lubricant shear velocity distribution during sliding. Fig 6 shows the layer-by-layer resolved ion density and velocity profiles along $z$ in the liquid for 7,5 and 3 layers, $F_{N}=0.5 \mathrm{nN}$ and $V=2.2 \mathrm{~m} / \mathrm{s} \mathrm{in}$ correspondence of green, blue and red symbols in Fig 5 a respectively. For $N_{\text {layer }}=7$ the velocity profile deviates from the linear behavior (red dashed line) indicating that the layers at the boundaries are bound to the plates and tend to move at their same velocity. The plate charging promotes the IL in immediate contact to nearly solid or at least to a much higher viscosity. Moreover, for this relatively high level of charging, the density ratio of first-layer cations over the anode, and of anions 
over the cathode, is about 1.4, that is only slightly larger than one. [14] Both elements support a good grip by the plates. The three inner layers constitute by contrast the more liquid, effective shear band where the sliding is concentrated, and where the coalescence between the two solid-like structures protruding from opposite plates is easiest to fracture. By reducing the number of layers to 5 and then to 3 , the shear band moves closer and closer to the boundaries and even the more solid-like structures closer to the plates must break up and move, as shown in Fig. 6d, f. As a consequence the lateral force $\left\langle F_{L}\right\rangle$ increases. A pictorial representation of this behavior is provided by the cartoons of Fig,6. Surprising at first sight is the seemingly "viscous" friction for $N_{\text {layer }}=3$, with a linear speed profile distribution between the three layers, the absence of strong stick-slip as seen in in Fig 4 a and, as we shall see later in Fig.9b, a linear friction increase with speed. That behavior coexists with a very large shear stress, and a very solid structure of this extremely thin lubricant film. The evolution of layer-bylayer relative velocities shown in Fig. 6 indicates that the yield stresses of the plate/IL interface and of the mid-film intra-IL interface, which for $N_{\text {layer }}=7,5$ were quite different, have now become comparable for $N_{\text {layer }}=3$. As a result all interfaces slide by about the same amount. The meandering wall $(x, y)$ static structure adopted by the IL in this pressured-and-charged regime [15] now incessantly ruptures and rearranges during sliding, leading to a z-uniform velocity gradient, reminscent of fluid lubrication, despite its nature very different from that of a fluid. The picture changes drastically by reducing the charge to $q=-4 \mu \mathrm{C} / \mathrm{cm}^{2}$ symmetrically on both plates. The mid-film shear band disappears and the slippage occurs mostly at the interfaces between the IL and the plates, as shown in Fig.7. While for $q=-12 \mu \mathrm{C} / \mathrm{cm}^{2}$ the top plate moved by rupturing the solid-like structures reaching across plates, at this lower charge the film behaves effectively as a solid block confined between two slippery surfaces. The shearing concentrates mostly at the two interfaces, whence the shear stress depends much more on the plate-IL interaction.

The frictional shear stress $\langle\sigma\rangle=\left\langle F_{L}\right\rangle / A$ is about an order of magnitude smaller than for higher charge and displays a much weaker dependence on $N_{\text {layer }}$, as seen by comparing Fig $8 \mathrm{~b}$ with Fig $\sqrt[5]{3}$. The rise of average frictional force $\left\langle F_{L}>\right.$ for $N_{\text {layer }}=3$, displayed in Fig 8 a (red line) is associated with a stick-slip regime, as shown by instantaneous spring forces in Fig 8 a corresponding to the points indicated in Fig $8 \mathrm{p}$. This is in contrast to the case of high charge, where friction was large but the sliding was smooth.

The essence for this difference of behavior is explained by the presence, at low but not at large plate charging, of overscreening, a phenomenon well demonstrated in earlier work [14]. At the small charging of $|q|=4 \mu \mathrm{C} / \mathrm{cm}^{2}$ we find that the charge density ratio of first-layer cations

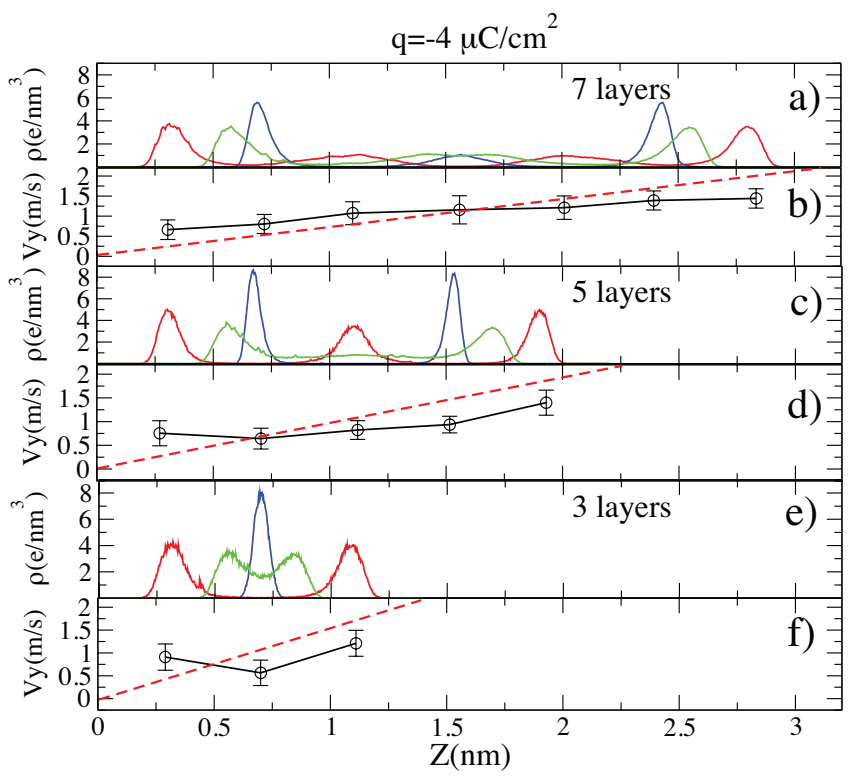

FIG. 7: Charge density $q=-4 \mu \mathrm{C} / \mathrm{cm}^{2}$ on both plates, top plate driven with velocity $V=2.2 \mathrm{~m} / \mathrm{s}$. Layer-by-layer density profiles ( (a), (c), (e) ) and sliding velocity ( (b), (d), (f) ) relative to the bottom plate, for $N_{\text {layer }}=7, N_{\text {layer }}=5$ and $N_{\text {layer }}=3$ respectively. Red, blue and green curves represent cation, anion and tail densities respectively. For this low charge density the sliding occurs mostly at interface between the IL film and the plates. Error bars in panels b, d, $f$ have been reduced ten times for clarity.

over that of the plate anode has in fact risen to about 2.3, much larger than one. The overscreened plate is covered by a dense counterion blanket, making the plate-IL adhesive grip quite effective. The IL film, on the other hand, is solid and even harder to fracture. The plate-IL interface yields under sliding and that causes stick-slip.

\section{VELOCITY DEPENDENCE OF FRICTION}

After the charge, load, and layer number dependence of confined IL friction, we close the symmetric plate charging part of this study by investigating the velocity dependence. A starting consideration here is that experimental sliding velocities in SFA and AFM are very small, typically reaching $10^{-6} \mathrm{~m} / \mathrm{s}$, a value which is at least 6 orders of magnitude lower than velocities realistically accessible in atomic and molecular level simulations. This is a standard difficulty, well known and amply discussed [34]; one can in fact learn enough even by simulating at very high speeds. Essentially, friction can be either smooth and viscous, in which case the shear stress is small and proportional to velocity, or it can be stick-slip, in which case the shear stress is large and very poorly dependent on velocity. In both cases, very crudely speaking, even a fast simulation can convey the necessary information 


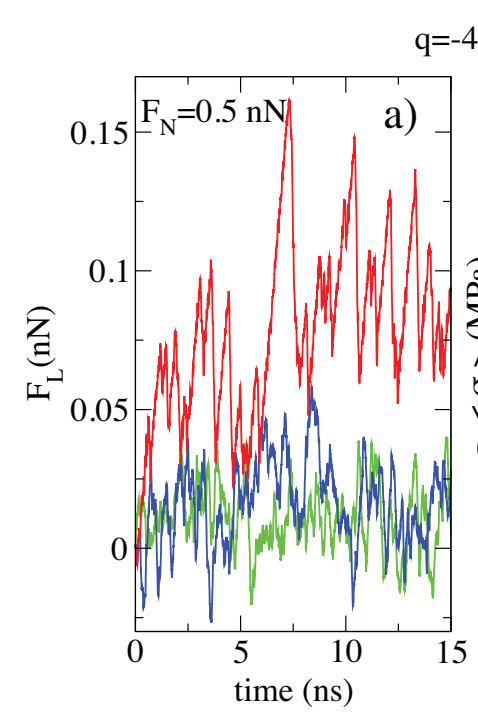

$\mathrm{q}=-4 \mu \mathrm{C} / \mathrm{cm}^{2}$

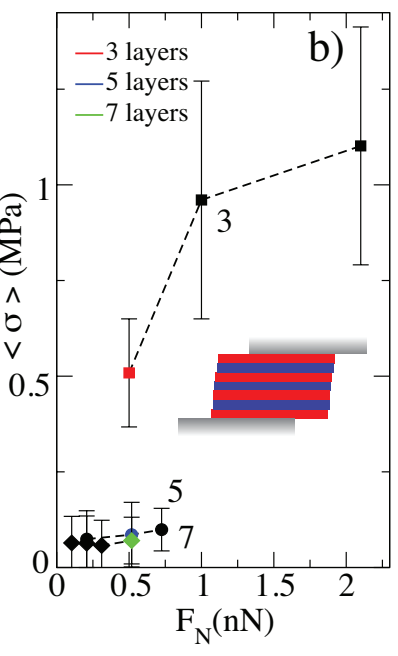

FIG. 8: a) Frictional force $F_{L}$ as a function of time for equal and low plate charge density $q=-4 \mu \mathrm{C} / \mathrm{cm}^{2}$, and for different number of confined layers $N_{\text {layer. }}$ b) Average shear stress $\sigma=\left\langle F_{L}>/ A\right.$ as a function of $F_{N}$ for indicated number of confined layers and $A=177 \mathrm{~nm}^{2}$. Colored markers refer to the time profiles shown in panel a). The cartoon shows that for this low charge density the slippage mostly occurs at interfaces between the liquid and plates.
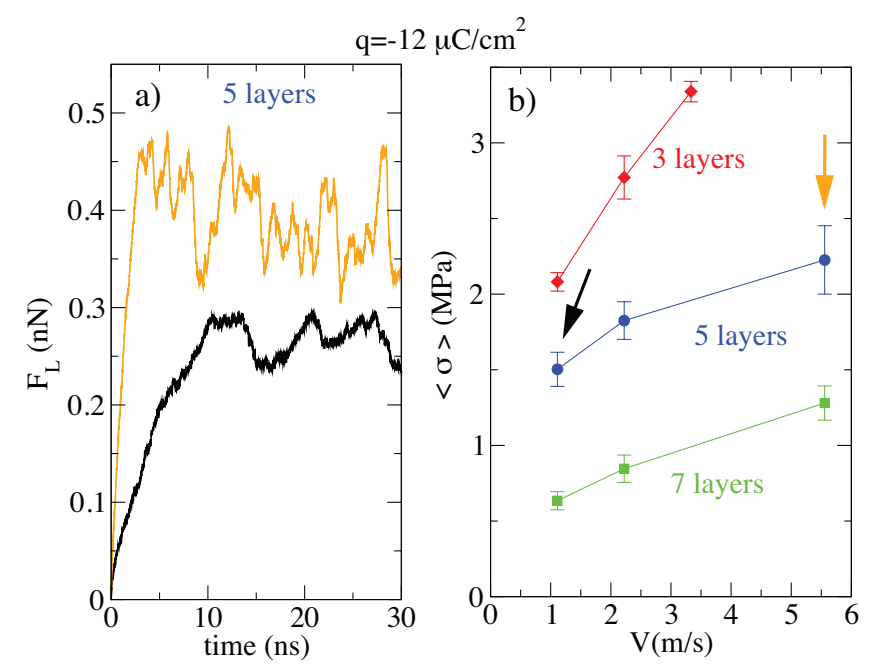

FIG. 9: a) Frictional force $F_{L}$ as a function of time for $N_{\text {layer }}=5, q=-12 \mu \mathrm{C} / \mathrm{cm}^{2}$ and velocities indicated by arrows in panel b. b) Average shear stress $\langle\sigma\rangle=\left\langle F_{L}\right\rangle / A$ as a function of driving velocity $V$ for different number of confined layers $N_{\text {layer }}=3,5,7$. The value of normal load here is $F_{N}=0.5 n N$.

(the viscous friction coefficient in the first, the full shear stress in the second). Of course it will always be necessary to bear in mind that a) increasing velocity may turn stick-slip into smooth sliding; and b) microscopic scale stick-slip does not necessarily show up as macroscopic or mesoscopic oscillations, and is generally detectable as a velocity independent shear stress; c) quantitative aspects are controlled by parameters such as the slider's masses, the pulling spring constant, etc., whose assumedvalues are largely arbitrary.

With these provisions we investigate the dependence of IL friction upon driving velocity $V$, at fixed normal load $F_{N}=0.5 n N$. Fig 9 and 10 show the frictional shear stress as a function of $V$ for equal-sign charge densities of $q=-12 \mu \mathrm{C} / \mathrm{cm}^{2}$ and $q=-4 \mu \mathrm{C} / \mathrm{cm}^{2}$ respectively and for $N_{\text {layer }}=3,5,7$. All results for $q=-12 \mu \mathrm{C} / \mathrm{cm}^{2}$ in Fig 9 show a linear increase of shear stress with velocity indicating a viscous-type friction. As suggested earlier, this is most likely attributable to overscreening of both plates by the extra anions. However $\langle\sigma\rangle$ vs $V$ does not extrapolate to 0 , so that even at $V \simeq 0$ a finite force is needed to set the system in motion. This is the force needed in order to rupture the vertical IL "solid" walls that straddle across the two charged plates (Fig 5).

Also for low charge density $q=-4 \mu \mathrm{C} / \mathrm{cm}^{2}$, the shear stress for $N_{\text {layer }}=5,7$ grows proportional to velocity but here it extrapolates to zero as $V \rightarrow 0$ (see Fig 10b). This is the hallmark of true viscous friction, and it agrees with the top plate moving in a clear regime of smooth sliding. The sliding regime drastically changes for $N_{\text {layer }}=3$ and low velocity $V=1.1 \mathrm{~m} / \mathrm{s}$, where a clear stick-slip regime takes over. The alternation of sticking periods followed by rapid and highly dissipative motion of the top plate is responsible for the strong increase of average lateral force at low velocities, as demonstrated in Fig 10 . By increasing $V$, the stick-slip first turns chaotic and eventually disappears at $V=5.5 \mathrm{~m} / \mathrm{s}$, where smooth sliding is recovered. Particle trajectories clearly show this transition. At low $V$ the IL structure retains its worm-like meandering planes or chains, which we described in ref. [15, and which were recently observed in experiments, [31] and behaves essentially as a solid confined between two slippery planes.

The transition from stick-slip to smooth sliding is shown in Fig 10 a, reporting two force profiles for $N_{\text {layer }}=3$ corresponding to driving velocities indicated by arrows in Fig 10 b.

\section{FRICTION WITH OPPOSITE PLATE CHARGING}

In this final part we study how friction force is affected by opposite charging of the two confining plates, such as one would obtain by applying a DC voltage between them. Probably not that easy to realize experimentally, this configuration is nonetheless quite interesting. Upon balancing the opposite charge on the two plates, the equilibrium layering structure now forms an even number of layers, a rearrangement which preserves local charge neutrality. In order to explore expeditely the dependence 


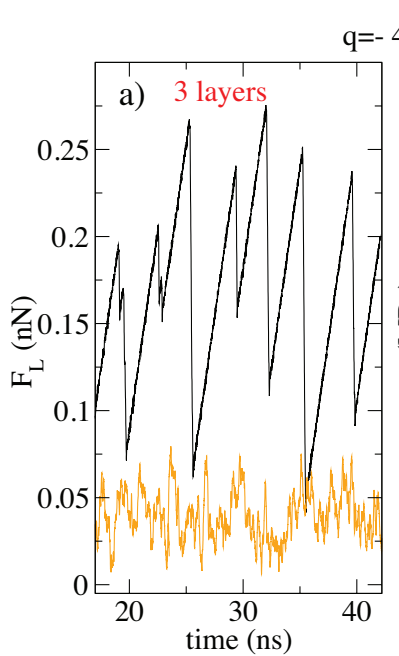

$$
\mathrm{q}=-4 \mu \mathrm{C} / \mathrm{cm}^{2}
$$

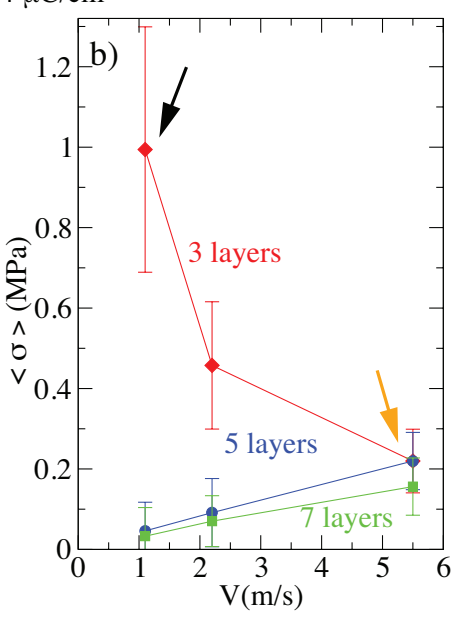

FIG. 10: a) Lateral force $F_{L}$ as a function of time for 3 layers, $q=-4 \mu \mathrm{C} / \mathrm{cm}^{2}$ and velocities indicated by arrows in panel b. b) Average shear frictional stress $\langle\sigma\rangle=\left\langle F_{L}\right\rangle / A$ as a function of driving velocity $V$ for different number of confined layers $N_{\text {layer }}$. The value of normal load here is $F_{N}=0.5 n N$.

of friction upon the magnitude of the charge imbalance, we conduct a time-dependent simulation where we periodically, but very slowly and as adiabatically as possible, modulate in time the on-plate charge in the form $q= \pm Q \sin (2 \pi t / \tau)$, where the two opposite signs refer to the two opposite plates. Adiabaticity is pursued by choosing a period $\tau$ large enough compared to the characteristic time scale of all relevant ionic rearrangements.

The magnitude of charging used in this work, $Q \leq$ $12 \mu \mathrm{C} / \mathrm{cm}^{2}$ is dictated, as discussed previously [15, by the requirement that, assuming an average dielectric constant of 2 , the plate-plate voltage should not exceed $5 \mathrm{~V}$, taken as a reasonable upper limit in an experimental situation. In this respect therefore, our study does not overlap with previous work, where in a different model 13 . the behavior at $Q \geq 16 \mu \mathrm{C} / \mathrm{cm}^{2}$ was investigated.

Assuming a constant load $F_{N}=1 \mathrm{nN}$, a charge oscillation period $\tau=72 \mathrm{~ns}$, a magnitude $Q=10 \mu \mathrm{C} / \mathrm{cm}^{2}$ and a sliding velocity $V=22 \mathrm{~m} / \mathrm{s}$ we obtain the spontaneous evolution of the system shown in Fig.11. What happens is, in a nutshell, that the interplate distance $D$ spontaneously changes, and so does the sliding friction $F_{L}$ as the charge changes. The simulation begins with a charge density of $|q(t=0)|=0$ on plates and the IL arranges in one single neutral layer indicated as $1 n$ in Fig 11, which, as shown in the static squeezout enthalpy of Fig.12, is the most stable configuration under the fixed applied loading force $F_{N}=1 \mathrm{nN}$ and low charge. As the plate charge increases, the liquid in the lateral reservoirs is sucked in the gap (despite a constant load), reaching a four layer solid state. Actually, we expected it to go back to six layers, which is the equilibrium free enthalpy minimum, as shown in Fig 12, It probably would if we could simu-

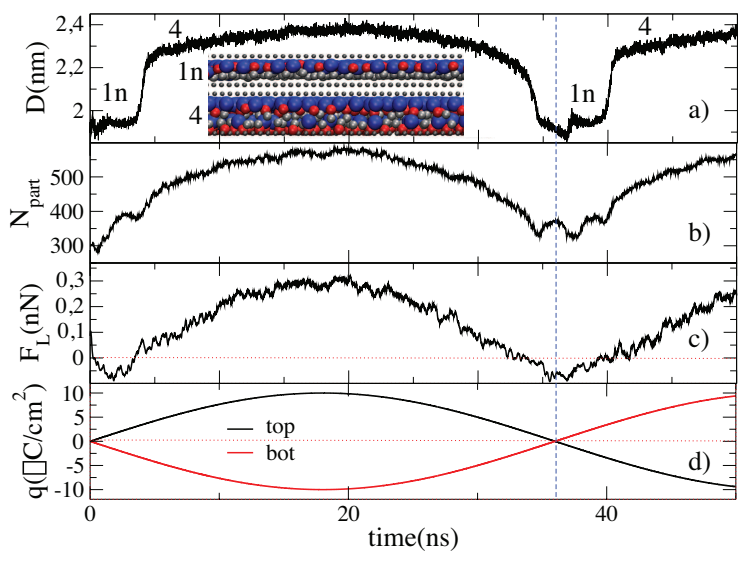

FIG. 11: a) Frictional sliding evolution while slowly changing opposite charges on the two sliding plates. Parameters: Initial $N_{\text {layer }}=1, F_{N}=1 \mathrm{nN}$, charges $q= \pm Q \sin (2 \pi t / \tau)$ with $Q=10 \mu \mathrm{C} / \mathrm{cm}^{2}$ and $\tau=72 \mathrm{~ns}$, sliding velocity $V=22 \mathrm{~m} / \mathrm{s}$. a) Spontaneous evolution of plate-plate distance $D$. b) Number of particles $N_{\text {part }}$ in the interplate gap. c) Sliding force $F_{L}$ as a function of time. d) Time evolution of top and bottom plate charge. Charging causes the plate separation $D$ to open up at charge maxima (IL suck-in) and to collapse at charge zeroes (IL squeezout), all under constant load. The inset in panel a) shows a side view of the confined liquid structure for 4 layers $(q=Q)$ and for the single neutral planar layer $1 n$ $(q=0)$. Friction is large when $|q|$ and voltage are maxima, and charging solidifies the IL inside the gap $D$. Conversely, when $q$ is near zero, the IL melts, the plates collapse together squeezing out all fluid except for a neutral monolayer where both ions pack in a square lattice, resulting in a very low friction.

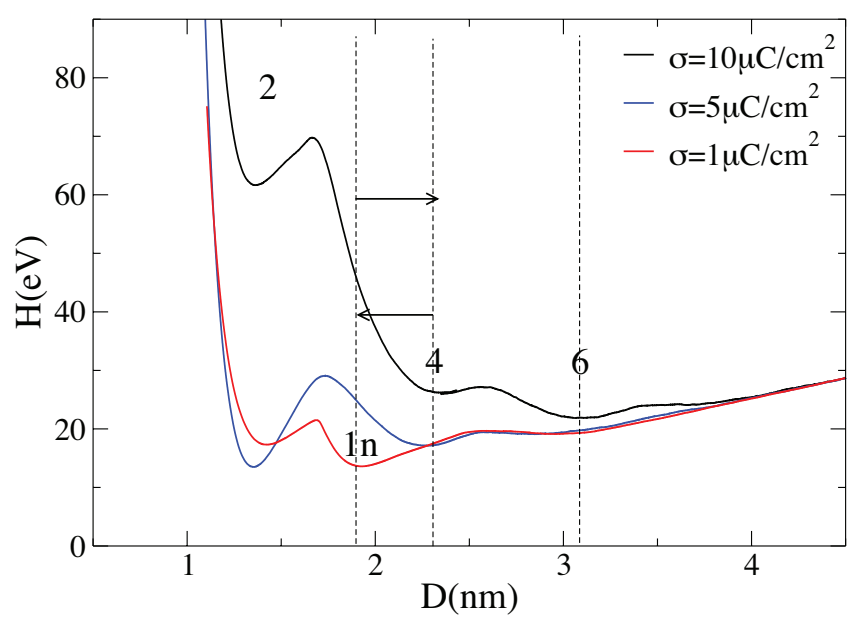

FIG. 12: (Color online) Enthalpy curves for indicated values of charge density on plates. Numbers indicated in figure denote metastable states at different number of layers. Peak heights decrease with charge and arrows indicate charge induced transitions between the states $1 n$ and 4 . 
late for much longer times, but it does not on our limited time scale, due to tha strongly reduced mobility of ions in the near-solid film. This strong and rigid four layers structure softens as the plate charge decreases, eventually turning more fluid and squeezing out reaching again the single neutral layer arrangement $1 n$. These charginginduced relayering and squeezout are accompanied by drastic change in frictional behaviour of the liquid film. The large shear stress at high plate charge (high voltage, near $5 \mathrm{eV}$ ) is replaced by a nearly vanishing shear stress at zero charge. Here therefore, contrary to the equalcharge case of the previous sections, the thinnest film yields the lowest friction, rather than the opposite. This is because, independently of sliding, the plate charging induces structural transformations of freezing/melting of the the IL film[15]. The frozen IL supports the applied load for large charge but the liquid IL does not for zero charge. The IL film structure at $q \simeq 0$ shrinks to a single, planar, square and neutral IL monolayer. Here the neutral tails stick out of the plane, effectively lubricating the sliding. A qualitatively similar mechanism where alkyl tails stick out of a well-packed anion-cation plane has been proposed in a recent experimental study [8] to explain the potential-dependent frictional behaviour of an AFM tip in presence of an IL.

We did change and explore a variety of parameters, including charging magnitude, applied load, and sliding speed in order to test the main aspects of these results. The processes of melting and freezing, and of expulsion and sucking always remained very asymmetrical between the fast squeezout, associated with sudden melting of the IL film upon decreasing $|q|$ towards zero, and the more gradual sucking-in and freezing, attained upon increasing $|q|$ from zero in Fig 11 . The electrically driven fast emptying but slow refilling of the interplate gap could be used, together with some spatial asymmetry to build an effective effective electro-pumping of ions in and out of the gap. The frictional shear stress shows a very large charge dependence, as a direct consequence of the changes of structure and of gap width $D(q)$ caused by charging. Although noisy, the large shear stress at charge maxima drops by an order of magnitude or more following squeezout at zero charge. This strong effect appears promising in view of experimental verifications, and of a possible use for the control of friction.

Lastly, we briefly dwell on questions connected with work, heat and temperature, which come about with respect both to charging-induced melting and freezing, and to frictional work and heating. In the absence of sliding, electrically induced freezing and melting would be associated with the absorption or release of latent heat. That would be large if the solid was ordered, e.g., crystalline or nearly crystalline, as is the case for example in the $\mathrm{NaCl}$ model of the IL 15 . The more the solid resembles a glass, however, the smaller the latent heat will be, owing to structural near-identity between glass and liq-

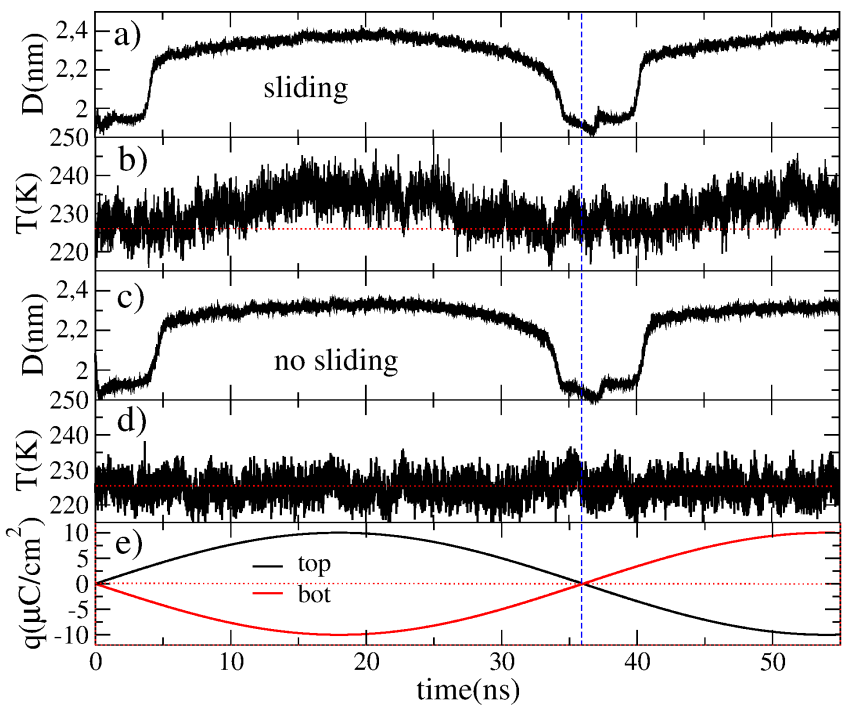

FIG. 13: (Color online) Comparison between the charge induced squeezout and relayering processes in the presence and absence of sliding. Spontaneous evolution of plate-plate distance $D((\mathrm{a})$ and (c)) and temperature ((b) and (d)) in comparison with time evolution of top and bottom plate charge (e). In the absence of driving the temperature remains stable at the set value of $T=225 \mathrm{~K}$, while in the presence of driving it temporarily deviates from this value.

uid. That is the case of our TM model, where indeed the latent heat of melting was very small in the simulated caloric curves [15]. Without sliding, the presence of a latent heat is undetectable in electrically induced freezing and melting cycles shown in Fig 13c,d. Of course, these data were obtained with a thermostat, canceling any large temperature fluctuations: despite the themostat, dips and peaks would be well visible in presence of a robust, crystal-like latent heat. Frictional heating is instead quite visible in the sliding results under cycled charging of Fig,13a,b. Despite the thermostat, the temperature is seen to oscillate, downwards when the solid confined IL lubricant goes liquid at $q=0$, up when it goes back to solid at $|q|=$ maximum and where therefore the frictional Joule heating is much stronger, down again upon melting where the friction drops once again. Because of the presence of the thermostat we did not attempt a quantitative connection between the frictional work and the residual temperature oscillation, but the connection between the two is quite clear.

\section{CONCLUSIONS}

The electrical charging of plates in nearly atomic contact influences an IL when trapped in a nanoscale sized gap between them. As a result, the frictional shear stress realized upon mutual sliding of the plates is modified by the charging. The present simulation study conducted 
within the simple "tailed model" previously developed for the IL explores some of the rich variety of phenomena that can take place in this context. The IL becomes structured into alternating charge layers, whose number is odd when the plates are equally charged, even when they are oppositely charged. The trapped IL film may develop a solid-like rigidity, with different and opposite frictional consequences depending on charging level and other parameters. When the plate grips the solid-like film and the shearing takes place in its middle the friction is large; but when the grip is less effective the shear concentrates at the plate-film interface and friction drops. Alternatively, at zero charging and under the same conditions the ionic liquid can melt, thus lubricating and dramatically reducing the sliding friction.

Even though the parameter-dependent nature of our results does not make a comparison with any specific experimental system particularly compelling, we believe that several elements uncovered either connect with observations already available, or else suggest pursuing newer ones. The modest dependence of friction upon load - unusual in the context of classical friction - is among the former. The possibility that electrical charging, in whichever manners that could be realized, could influence the tribology of confined IL by causing its electrically driven freezing and melting is among the latter, and deserves to be tested, supporting the possibility of an electric control of IL lubricated friction.

\section{ACKNOWLEDGMENTS}

The authors are grateful to Daniele Passerone and Carlo Pignedoli from EMPA (Dübendorf, $\mathrm{CH}$ ) for the computational resources and the technical assistance provided, and, at various times, for discussions with A. Kornyshev and A. Schirmeisen. Work in Trieste was partly sponsored by Sinergia Contract CRSII2 $2_{1} 36287 / 1$, and by ERC Advanced Grant 320796 - MODPHYSFRICT. This work is also supported by the COST Action MP1303 "Understanding and Controlling Nano and Mesoscale Friction".

[1] N. V. Plechkova and K. R. Seddon, Chem. Soc. Rev. 37, 123 (2008).

[2] J. P. Hallett and T. Welton, Chem. Rev. 111, 3508 (2011).

[3] R. Hayes, G. G. Warr, and R. Atkin, Phys. Chem. Chem. Phys. 12, 1709 (2010).

[4] S. Perkin, T. Albrecht, and J. Klein, Phys. Chem. Chem. Phys. 12, 1243 (2010).

[5] R. M. Espinosa-Marzal, A. Arcifa, A. Rossi, and N. D. Spencer, J. Phys. Chem. Lett. 5, 179 (2014).

[6] O. Werzer, E. D. Cranston, G. G. Warr, R. Atkin, and M. Rutland, Phys. Chem. Chem. Phys. 14, 5147 (2012).
[7] H. Li, M. W. Rutland, and R. Atkin, Phys. Chem. Chem. Phys. 15, 14616 (2013).

[8] J. Sweeney, F. Hausen, R. Hayes, G. B. Webber, F. Endres, M. W. Rutland, R. Bennewitz, and R. Atkin, Phys. Rev. Lett. 109, 155502 (2012).

[9] H. Li, R. J. Wood, M. W. Rutland, and R. Atkin, Chem. Commun. 50, 4368 (2014).

[10] J. Hoth, F. Hausen, M. H. Müser, and R. Bennewitz, J. Phys.: Condens. Matter 26, 284110 (2014).

[11] A. Mendoncą, A. Pàdua, and P. Malfreyt, J. Chem. Theory Comput. 9, 1600 (2013).

[12] F. Federici-Canova, H. Matsubara, M. Mizukami, K. Kurihara, and A. L. Shluger, Phys. Chem. Chem. Phys. 16, 8247 (2014).

[13] O. Fajardo, F. Bresme, A. Kornyshev, and M. Urbakh, Sci. Rep. 5, 7698 (2015).

[14] M. Fedorov and A. Kornyshev, J. Phys. Chem. B 112, 11868 (2008).

[15] R. Capozza, A. Vanossi, A. Benassi, and E. Tosatti, J. Chem. Phys. 142, 064707 (2015).

[16] T. Y. Kim, H. W. Lee, M. Stoller, D. R. Dreyer, C. W. Bielawski, R. S. Ruoff, and K. S. Suh, ACS Nano 5, 436 (2011).

[17] Y. Shim and H. J. Kim, ACS Nano 4, 2345 (2010).

[18] J. Black, D. Walters, A. Labuda, G. Feng, P. Hillesheim, S. Dai, P. Cummings, S. Kalinin, R. Proksch, and N. Balke, Nano Lett. 13, 5954 (2013).

[19] C. Dold, T. Amann, and A. Kailer, Phys. Chem. Chem. Phys. 17, 10339 (2015).

[20] X. Yang, Y. Meng, and Y. Tian, Trib. Lett. 56, 161 (2014).

[21] A. Smith, K. Lovelock, N. Gosvami, T. Welton, and S. Perkin, Phys. Chem. Chem. Phys. 15, 15317 (2013).

[22] R. Atkin and G. G. Warr, J. Phys. Chem. C 111, 5162 (2007).

[23] I. Bou-Malham and L. Bureau, Soft Matter 6, 4062 (2010).

[24] K. Ueno, M. Kasuya, M. Watanabe, M. Mizukami, and K. Kurihara, Phys. Chem. Chem. Phys. 12, 4066 (2010).

[25] S. Perkin, L. Crowhurst, H. Niedermeyer, T. Welton, A. M. Smith, and N. N. Gosvami, Chem. Commun. 47, 6572 (2011).

[26] S. Perkin, Phys. Chem. Chem. Phys. 14, 5052 (2012).

[27] H. Li, F. Endres, and R. Atkin, Phys. Chem. Chem. Phys. 15, 14624 (2013).

[28] A. M. Smith, K. R. J. Lovelock, N. N. Gosvami, P. Licence, A. Dolan, T. Welton, and S. Perkin, J. Phys. Chem. Lett. 4, 378 (2013).

[29] S. Plimpton, J. Comp. Phys. 117, 1 (1995).

[30] B. L. D. Northern, Y. L. Chen, J. N. Israelachvili, and J. A. N. Zasadzinski, Proceedings of the 49th Annual Meeting of the Electron Microscopy Society of America, Atomic force microscopy of mica surface after ion replacement, p.628 (San Francisco Press., Inc., San Francisco, CA 94101-6800, USA, 1991).

[31] J. J. Segura, A. Elbourne, E. J. Wanless, G. G. Warr, K. Voïchovsky, and R. Atkin, Phys. Chem. Chem. Phys. 15, 3320 (2013).

[32] A. Elbourne, K. Voïchovsky, G. G. Warr, and R. Atkin, Chem. Sci. 6, 527 (2015).

[33] R. Hayes, N. Borisenko, M. Tam, P. Howlett, F. Endres, and R. Atkin, J. Phys. Chem. C 115, 6855 (2011).

[34] A. Vanossi, N. Manini, M. Urbakh, S. Zapperi, and E. Tosatti, Rev. Mod. Phys. 85, 529 (2013). 